\title{
COMMUTATIVITY DEGREES OF WREATH PRODUCTS OF FINITE ABELIAN GROUPS
}

\author{
IGOR V. EROVENKO and B. SURY
}

\author{
(Received 12 April 2007)
}

\begin{abstract}
We compute commutativity degrees of wreath products $A$ < $B$ of finite Abelian groups $A$ and $B$. When $B$ is fixed of order $n$ the asymptotic commutativity degree of such wreath products is $1 / n^{2}$. This answers a generalized version of a question posed by P. Lescot. As byproducts of our formula we compute the number of conjugacy classes in such wreath products, and obtain an interesting elementary numbertheoretic result.
\end{abstract}

2000 Mathematics subject classification: 20E22, $20 \mathrm{~J} 06$.

Keywords and phrases: wreath product, commutativity degree.

\section{Introduction}

For a finite group $G$ let $\mathcal{G}$ denote the set of pairs of commuting elements of $G$ :

$$
\mathcal{G}=\{(g, h) \in G \times G \mid g h=h g\} .
$$

The quantity $|\mathcal{G}| /|G|^{2}$ measures the probability of two random elements of $G$ commuting and is called the commutativity degree of $G$. In [1] Lescot computes the commutativity degree of dihedral groups and shows that it tends to $1 / 4$ as the order of the group tends to infinity. He then asks whether there are other natural families of groups with the same property. In this paper we show that if $B$ is an Abelian group of order $n$ and $A$ is a finite Abelian group, then the commutativity degree of the wreath product $A$ < $B$ tends to $1 / n^{2}$ as the order of $A$ tends to infinity.

Theorem 1.1. Let $G=A 々 B$, where $A$ is a finite Abelian group and $B=\left\{b_{1}, b_{2}, \ldots, b_{n}\right\}$ is an Abelian group of order $n$. Then

$$
|\mathcal{G}|=\sum_{s, t=1}^{n}|A|^{n+\alpha(s, t)},
$$

where $\alpha(s, t)$ denotes the index of the subgroup of $B$ generated by $b_{s}$ and $b_{t}$.

(C) 2008 Australian Mathematical Society 0004-9727/08 \$A2.00+0.00 
The exact value of the quantity $\alpha(s, t)$, of course, depends on the structure of $B$ as an Abelian group. We show how to obtain it in Section 3. Here we just note that when $B=\mathbb{Z}_{n}=\{1,2, \ldots, n\}$ is a cyclic group of order $n, \alpha(s, t)=(n, s, t)$ (where $(n, s, t)$ denotes the greatest common divisor of $n, s$, and $t)$. More generally, for a fixed value of $n$ the farther $B$ is away from a cyclic group, the larger the commutativity degree of the wreath product $A$ ? $B$ is. For example, the commutativity degree of $A \geq \mathbb{Z}_{4}$ is $1 / 16+3|A|^{-2}+12|A|^{-3}$, while that of $A$ ? $\left(\mathbb{Z}_{2} \times \mathbb{Z}_{2}\right)$ is $1 / 16+9|A|^{-2}+6|A|^{-3}$. However, the asymptotic behaviour of the commutativity degree of the wreath product $A$ 乙 $B$ as $|A| \rightarrow \infty$ does not depend on the structure of $B$ as an Abelian group.

COROllary 1.2. Let $A$ be a finite Abelian group and $B$ be an Abelian group of order $n$. Then the commutativity degree of the wreath product $A$ ? $B$ tends to $1 / n^{2}$ as $|A| \rightarrow \infty$.

A straightforward computation with indices of centralizers shows that the number of conjugacy classes in a finite group $G$ is equal to $|\mathcal{G}| /|G|$, hence (1) yields the formula for the number of conjugacy classes in wreath products of finite Abelian groups.

Corollary 1.3. Let $A$ and $B$ be as in Theorem 1.1. Then the number of conjugacy classes in the wreath product $A$ ? $B$ is $(1 / n) \sum_{s, t=1}^{n}|A|^{\alpha(s, t)}$.

By taking $B=\mathbb{Z}_{n}$ in Corollary 1.3 , we obtain the following interesting elementary number-theoretic result. We have not been able to find an elementary proof of this fact.

COROLLARY 1.4. For any natural number $a$, the sum $\sum_{s, t=1}^{n} a^{(n, s, t)}$ is divisible by $n$. If $n$ is prime, this gives Fermat's little theorem.

\section{Notation and terminology for wreath products}

We shall use some of the notation from [2]. Let $A$ and $B$ be groups and let $A^{*}$ be the direct sum of copies of $A$ indexed by elements of $B$. We shall write this as $A^{*}=\sum_{b \in B} A_{b}$, where each group $A_{b}$ is a copy of $A$. Elements of $A^{*}$ can be thought of as functions from $B$ to $A$ with finite support. An element $f \in A^{*}$ such that

$$
f(b)= \begin{cases}a & \text { if } b=b_{0} \in B \\ e_{A} & \text { otherwise }\end{cases}
$$

will be denoted by $\sigma_{a}\left(b_{0}\right)$. In this notation, every element of $A^{*}$ can be uniquely written in the form

$$
\sigma_{a_{1}}\left(b_{1}\right) \cdots \sigma_{a_{s}}\left(b_{s}\right),
$$

where $b_{1}, \ldots, b_{s}$ are distinct elements of $B$, and $a_{1}, \ldots, a_{s}$ are any elements of $A$. Such a presentation will be called a canonical word. Define an action of $B$ on $A^{*}$ by

$$
f^{c}(b)=f\left(b c^{-1}\right), \quad c \in B, b \in B .
$$


The (standard restricted) wreath product of $A$ and $B$, denoted by $A<B$, is the semidirect product of $A^{*}$ and $B$ with the action of $B$ on $A^{*}$ given by (2). If we denote the elements of the canonical copy of $B$ in $A$ < $B$ by $\tau_{c}, c \in B$, then (2) becomes

$$
\tau_{c} \sigma_{a}(b)=\sigma_{a}(b c) \tau_{c}
$$

and thus every element of $A$ \B can be uniquely written in the canonical form

$$
\sigma_{a_{1}}\left(b_{1}\right) \cdots \sigma_{a_{s}}\left(b_{s}\right) \tau_{b}
$$

where $\sigma_{a_{1}}\left(b_{1}\right) \cdots \sigma_{a_{s}}\left(b_{s}\right)$ is a canonical word in $A^{*}$. We shall work with wreath products where the group $B$ is finite, in which case the restricted wreath product and the complete wreath product are the same.

\section{Proof of Theorem 1.1}

Since both groups $A$ and $B$ are Abelian we shall use additive notation for their group operations. To make the proof transparent we first work out in detail the case when $B=\mathbb{Z}_{n}$ is the cyclic group of order $n$. We may represent elements of $B$ by arbitrary integers assuming that one takes the residue modulo $n$ to obtain an actual element of $\mathbb{Z}_{n}$.

We shall count the number of commuting pairs of elements of $G=A$ 々 $\mathbb{Z}_{n}$ as follows. Fix $s$ and $t$ in $\{1, \ldots, n-1, n\}$ and let

$$
g=\sigma_{a_{0}}(0) \sigma_{a_{1}}(1) \cdots \sigma_{a_{n-1}}(n-1) \tau_{-s},
$$

and

$$
h=\sigma_{x_{0}}(0) \sigma_{x_{1}}(1) \cdots \sigma_{x_{n-1}}(n-1) \tau_{-t} .
$$

We then count the number of commuting pairs $(g, h)$ with prescribed values of $s$ and $t$ but allowing the $a_{i}$ and $x_{i}$ to be arbitrary elements of $A$. To do so we think of an element $g$ as being 'fixed' and count the number of elements $h$ that commute with every such given $g$. As we shall see shortly, there might be some conditions on the $a_{i}$ for $g$ to commute with at least one such $h$.

We shall make a convention that $a_{u}$ and $a_{v}$ represent the same element of the group $A$ if $u$ and $v$ are equal modulo $n$; similarly for $x_{u}$ and $x_{v}$. With this notation, the elements $g$ and $h$ as above commute if and only if

$$
\begin{aligned}
x_{0}-x_{s} & =a_{0}-a_{t}, \\
x_{1}-x_{s+1} & =a_{1}-a_{t+1}, \\
& \vdots \\
x_{n-1}-x_{s+(n-1)} & =a_{n-1}-a_{t+(n-1)},
\end{aligned}
$$


which can be thought of as a 'linear system' in unknowns $x_{0}, x_{1}, \ldots, x_{n-1}$. Let $d+1$ be the order of $s$ in $\mathbb{Z}_{n}$, then $d+1=n /(n, s)$ and there are $(n, s)$ cosets of the cyclic subgroup $\langle s\rangle$ generated by $s$ in $\mathbb{Z}_{n}$.

The above linear system will split into $(n, s)$ independent subsystems in unknowns $\left\{x_{i}, x_{i+s}, x_{i+2 s}, \ldots, x_{i+d s}\right\}$ where $i$ varies over the representatives of the cosets of $\langle s\rangle$ in $\mathbb{Z}_{n}$, say $0 \leqslant i \leqslant(n, s)-1$. The matrix of each such subsystem has rank $d$; hence for the subsystem to be consistent the 'constant' column consisting of differences of $a_{i}$ must add up to zero. This gives the following condition for consistency of the $i$ th subsystem:

$$
\begin{array}{r}
a_{i}+a_{i+s}+\cdots+a_{i+d s}=a_{i+t}+a_{i+s+t}+\cdots+a_{i+d s+t}, \\
0 \leqslant i \leqslant(n, s)-1 .
\end{array}
$$

If $t \in\langle s\rangle$ then the conditions (3) are automatically satisfied for all $i$, and hence for any choice of the elements $a_{0}, a_{1}, \ldots, a_{n-1}$ the number of elements $h$ commuting with given $g$ is $|A|^{(n, s)}$ since each subsystem has one free variable.

Suppose now that $t \in j+\langle s\rangle$ for some $j \in\{1, \ldots,(n, s)-1\}$. Let $u$ denote the order of $t$ (= order of $j$ ) in the quotient group $\mathbb{Z}_{n} /\langle s\rangle$. Then $u=(n, s) /(n, s, t)$ and the index of the subgroup $\langle t\rangle$ in $\mathbb{Z}_{n} /\langle s\rangle$ is $(n, s) / u=(n, s, t)$; in the notation of Theorem 1.1 this is nothing but $\alpha(s, t)$.

The conditions (3) split into $\alpha(s, t)$ blocks corresponding to the cosets of $\langle t\rangle$ in $\mathbb{Z}_{n} /\langle s\rangle$. The $k$ th block $(0 \leqslant k \leqslant \alpha(s, t)-1)$ looks as follows:

$$
\begin{aligned}
a_{k}+a_{k+s}+\cdots+a_{k+d s} & =a_{k+t}+a_{k+t+s}+\cdots+a_{k+t+d s}, \\
a_{k+t}+a_{k+t+s}+\cdots+a_{k+t+d s} & =a_{k+2 t}+a_{k+2 t+s}+\cdots+a_{k+2 t+d s}, \\
& \vdots \\
a_{k+(u-1) t}+a_{k+(u-1) t+s}+\cdots+a_{k+(u-1) t+d s} & =a_{k+u t}+a_{k+u t+s}+\cdots+a_{k+u t+d s} .
\end{aligned}
$$

But $u t$ is a multiple of $s$, and hence the right-hand side of the last equation is equal to the left-hand side of the first equation. It follows that exactly one of these $u$ equations is a consequence of the others and each block produces $u-1$ independent 'linear' conditions on the $a_{i}$.

To summarize, among the $|A|^{n}$ sequences $\left(a_{0}, a_{1}, \ldots, a_{n-1}\right)$ of elements of $A$, there are exactly $|A|^{n-\alpha(s, t)(u-1)}=|A|^{n-(n, s)+\alpha(s, t)}$ sequences for which the original linear system in $x_{0}, x_{1}, \ldots, x_{n-1}$ is consistent. For each such fixed sequence, the number of sequences $\left(x_{0}, x_{1}, \ldots, x_{n-1}\right)$ satisfying the corresponding system is $|A|^{(n, s)}$ since each of the $(n, s)$ (= index of the subgroup of $B$ generated by $s$ ) subsystems contributes one free variable. Thus, for fixed $s$ and $t$ the total number of commuting pairs $(g, h)$ of elements of $G$ where the canonical form of $g$ ends in $\tau_{-s}$ and the canonical form of $h$ ends in $\tau_{-t}$ is $|A|^{n+\alpha(s, t)}$. The formula (1) now follows.

In the general case, when $B=\left\{b_{1}, b_{2}, \ldots, b_{n}\right\}$ is an arbitrary Abelian group, fix $b_{s}, b_{t} \in B$ and consider two elements of $G=A$ 々 $B$,

$$
g=\sigma_{a_{1}}\left(b_{1}\right) \sigma_{a_{2}}\left(b_{2}\right) \cdots \sigma_{a_{n}}\left(b_{n}\right) \tau_{-b_{s}}
$$


and

$$
h=\sigma_{x_{1}}\left(b_{1}\right) \sigma_{x_{2}}\left(b_{2}\right) \cdots \sigma_{x_{n}}\left(b_{n}\right) \tau_{-b_{t}} .
$$

Note that the above proof essentially did not use the fact that $B$ was a cyclic group (it was only used so as to have a convenient way to label the indices of $a_{i}$ and $x_{i}$ ). Rather, the computation involves the following quantities:

(1) the index of the cyclic subgroup of $B$ generated by $b_{s}$, say $\beta(s)$;

(2) the index of the cyclic subgroup of the quotient group $B /\left\langle b_{s}\right\rangle$ generated by the image of $b_{t}$, which is precisely $\alpha(s, t)$ in our notation.

The 'linear system' which gives conditions for elements $g$ and $h$ to commute then splits into $\beta(s)$ subsystems each of which corresponds to a coset of the cyclic subgroup $\left\langle b_{s}\right\rangle$ of $B$, and hence the same reasoning carries over verbatim to the general case. Further, the conditions on the $a_{i}$ will split into $\alpha(s, t)$ blocks each of which corresponds to a coset of the cyclic subgroup generated by the image of $b_{t}$ in $B /\left\langle b_{s}\right\rangle$.

It follows that among the $|A|^{n}$ sequences $\left(a_{1}, a_{2}, \ldots, a_{n}\right)$ of elements of $A$, there are exactly $|A|^{n-\beta(s)+\alpha(s, t)}$ sequences for which the linear system is consistent. For each such fixed sequence, the number of sequences $\left(x_{1}, x_{2}, \ldots, x_{n}\right)$ satisfying the corresponding system is $|A|^{\beta(s)}$. Thus, for fixed $s$ and $t$ the total number of commuting pairs $(g, h)$ of elements of $G$ where the canonical form of $g$ ends in $\tau_{-b_{s}}$ and the canonical form of $h$ ends in $\tau_{-b_{t}}$ is $|A|^{n+\alpha(s, t)}$. This completes the proof of Theorem 1.1.

Finally, we give a formula for $\alpha(s, t)$ which depends on the structure of $B$ as an Abelian group. Let $B=\mathbb{Z}_{n_{1}} \times \cdots \times \mathbb{Z}_{n_{k}}$ and let $s=\left(s_{1}, \ldots, s_{k}\right), t=\left(t_{1}, \ldots, t_{k}\right)$ be two elements of $B$. Let $\alpha(s, t)=[B:\langle s, t\rangle]$.

Consider the surjective homomorphism $\pi: \mathbb{Z}^{k} \rightarrow B$ with

$$
\operatorname{ker} \pi=n_{1} \mathbb{Z} \times \cdots \times n_{k} \mathbb{Z} \text {. }
$$

Let $a, b \in \mathbb{Z}^{k}$ be such that $\pi(a)=s$ and $\pi(b)=t$. Then $\mathbb{Z}^{k} / H \cong B /\langle s, t\rangle$ where $H=$ ker $\pi+\langle a, b\rangle$. We determine the order of $\mathbb{Z}^{k} / H$ as follows. Write $a=\left(a_{1}, \ldots, a_{k}\right)$ and $b=\left(b_{1}, \ldots, b_{k}\right)$ (thinking of the $s_{i}$ and $t_{j}$ as integers one may take $a_{i}=s_{i}$ and $b_{j}=t_{j}$ for all $\left.i, j \in\{1, \ldots, k\}\right)$, then

$$
H=\left\{\left(n_{1} m_{1}+u a_{1}+v b_{1}, \ldots, n_{k} m_{k}+u a_{k}+v b_{k}\right) \mid m_{i}, u, v \in \mathbb{Z}\right\} .
$$

If $R: \mathbb{Z}^{k+2} \rightarrow \mathbb{Z}^{k}$ is a homomorphism given by the $k \times(k+2)$ matrix

$$
\left[\begin{array}{cccccc}
n_{1} & 0 & \cdots & 0 & a_{1} & b_{1} \\
0 & n_{2} & \cdots & 0 & a_{2} & b_{2} \\
\vdots & \vdots & \ddots & \vdots & \vdots & \vdots \\
0 & 0 & \cdots & n_{k} & a_{k} & b_{k}
\end{array}\right]
$$

then $H=\operatorname{Im} R$. Let $P \in G L_{k}(\mathbb{Z})$ and $Q \in G L_{k+2}(\mathbb{Z})$ be such that 


$$
P R Q=\left[\begin{array}{cccccc}
d_{1} & 0 & \cdots & 0 & 0 & 0 \\
0 & d_{2} & \cdots & 0 & 0 & 0 \\
\vdots & \vdots & \ddots & \vdots & \vdots & \vdots \\
0 & 0 & \cdots & d_{k} & 0 & 0
\end{array}\right]
$$

where $d_{1}\left|d_{2}\right| \cdots \mid d_{k}$ are the elementary divisors of $R$. We have

$$
\mathbb{Z}^{k} / \operatorname{Im} R \cong P\left(\mathbb{Z}^{k}\right) / P R\left(\mathbb{Z}^{k+2}\right)=\mathbb{Z}^{k} / P R Q\left(\mathbb{Z}^{k+2}\right),
$$

so that

$$
\alpha(s, t)=\left|\mathbb{Z}^{k} / \operatorname{Im} R\right|=\left|d_{1} d_{2} \cdots d_{k}\right| .
$$

For the reader's convenience we recall a well-known method for finding elementary divisors. For $i=1, \ldots, k$, let $h_{i}$ denote the greatest common divisor of all $i \times i$ minors of $R$; then $h_{i}=d_{1} d_{2} \cdots d_{i}$. This is because the numbers $h_{i}$ do not change when multiplied on the left and on the right by elementary matrices and these generate all invertible integer matrices. In particular, note that if $k=1$ then $\alpha(s, t)=(n, s, t)$.

\section{Acknowledgement}

The second author would like to thank the Max Planck Institut für Mathematik for its hospitality during his visit in February-March 2007 when this paper was written.

\section{References}

[1] P. Lescot, 'Central extensions and commutativity degree', Comm. Algebra 29 (2001), 4451-4460.

[2] J. D. P. Meldrum, Wreath products of groups and semigroups, Pitman Monographs and Surveys in Pure and Applied Mathematics, 74 (Longman, Harlow, 1995).

Department of Mathematics and Statistics University of North Carolina at Greensboro

Greensboro, NC 27402

USA

e-mail: igor@uncg.edu
Stat-Math Unit

Indian Statistical Institute 8th Mile Mysore Rd Bangalore 560059 India e-mail: sury@isibang.ac.in 